\title{
Editorial
}

\section{Theory and Applications of Fractional Order Systems}

\author{
Riccardo Caponetto, ${ }^{1}$ Josè A. Tenereiro Machado, ${ }^{2}$ and Juan J. Trujillo ${ }^{3}$ \\ ${ }^{1}$ Department of Electrical, Electronics and Computer Engineering, University of Catania, Viale A. Doria 6, 95125 Catania, Italy \\ ${ }^{2}$ ISEP-Institute of Engineering, Polytechnic of Porto, Department of Electrical Engineering, Rua Dr. Antonio \\ Bernardino de Almeida 431, 4200-072 Porto, Portugal \\ ${ }^{3}$ Department of Mathematical Analysis, University of La Laguna, C/Astr. Francisco Sanchez s/n, La Laguna, 38271 Tenerife, Spain
}

Correspondence should be addressed to Riccardo Caponetto; riccardo.caponetto@dieei.unict.it

Received 16 November 2014; Accepted 16 November 2014; Published 30 December 2014

Copyright (C) 2014 Riccardo Caponetto et al. This is an open access article distributed under the Creative Commons Attribution License, which permits unrestricted use, distribution, and reproduction in any medium, provided the original work is properly cited.

In the last decades noninteger differentiation became a popular tool for modeling the complex behaviours of physical systems from diverse domains such as mechanics, electricity, chemistry, biology, and economics. Numerous studies have validated the novel perspective demonstrating fractional order models that better characterize many real-world physical systems by means of differential operators of noninteger order. The long-range temporal or spatial dependence phenomena inherent to the fractional order systems (FOS) present unique and intriguing peculiarities, not supported by their integer-order counterpart, which raise exciting challenges and opportunities related to the development of modelling, control, and estimation methodologies involving fractional order dynamics.

The purpose of this special issue is to draw attention to the scientific community to some recent advances and possible applications of fractional order systems and to ensure the corresponding dissemination. The issue includes a collection of papers in the area of FOS and some leading and emerging specialists in the area present their latest results.

A short description of the addressed topics is as follows.

(i) Forest fires are studied in the perspective of dynamical systems, describing the global dynamics along several decades. The time is modelled as Dirac impulses with amplitude proportional to the burnt area.

(ii) A systematic form of the existing formulations of fractional derivatives and integrals is presented. (iii) The asymptotic stability of the two-step RungeKutta methods for neutral delay integrodifferentialalgebraic equations with many delays is developed. It has been proved that A-stable two-step RungeKutta methods are asymptotically stable for neutral delay integrodifferential-algebraic equations with many delays.

(iv) An efficient iteration method for Toeplitz-plus-band triangular systems is presented with $O(M \log (M))$ computational complexity and $O(M)$ memory complexity. The proposed method is compared with the regular solution with $\left(M^{2}\right)$ computational complexity and $O\left(M^{2}\right)$ memory complexity.

(v) The fundamental solutions to time-fractional advection diffusion equation in a plane and a half-plane are obtained using the Laplace integral transform with respect to time $t$ and the Fourier transforms with respect to the space coordinates $x$ and $y$. The Cauchy, source, and Dirichlet problems are also investigated.

(vi) A novel watermarking method associated with the linear canonical transform is proposed. The linear canonical transform, which can be looked at as the generalization of the fractional Fourier transform and the Fourier transform, has received much interest and proved to be one of the most powerful tools in fractional signal processing community.

(vii) A finite series representation of the inverse MittagLeffler function is formulated for a range of the 
parameters $\alpha$ and $\beta$, specifically, $0<\alpha<1 / 2$ for $\beta=1$ and for $\beta=2$, showing also that this finite series representation of the inverse Mittag-Leffler function greatly expedites its evaluation.

(viii) The sparse prior in fractional order gradient domain as texture-preserving strategy to restore textured images degraded by blur and/or noise is introduced. The unknown variables in proposed model using method based on half-quadratic splitting by minimizing the nonconvex energy functional are also solved.

(ix) A new general and systematic coupling scheme is developed to achieve the modified projective synchronization (MPS) of different fractional order systems under parameter mismatch via the open-plusclosed-loop (OPCL) control. Based on the stability theorem of linear fractional order systems, sufficient conditions for MPS are proposed.

(x) Several nanodiamond preparations for Raman spectroscopic studies have been studied. These nanodiamonds have been exposed to increasing temperature treatments at constant heating rates $\left(425-575^{\circ} \mathrm{C}\right)$ aiding graphite release. Changes in the nanodiamond surface and properties with Raman signal which could be used as a detection marker are correlated.

(xi) The discrete wavelet transform via local fractional operators is structured and applied to process the signals on Cantor sets. An illustrative example of the local fractional discrete wavelet transform is also given.

Riccardo Caponetto Josè A. Tenereiro Machado Juan J. Trujillo 


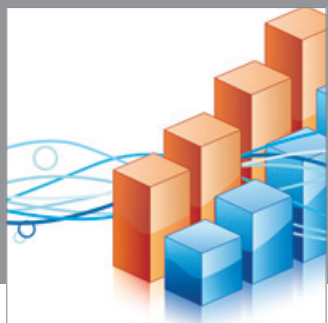

Advances in

Operations Research

mansans

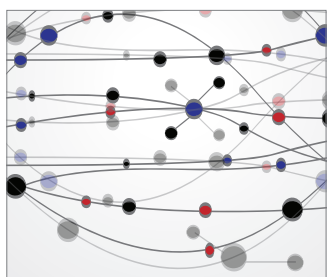

The Scientific World Journal
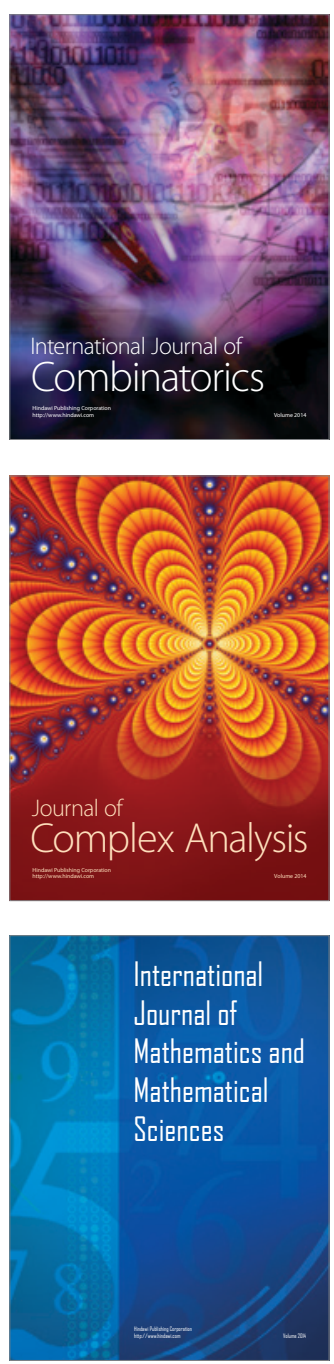
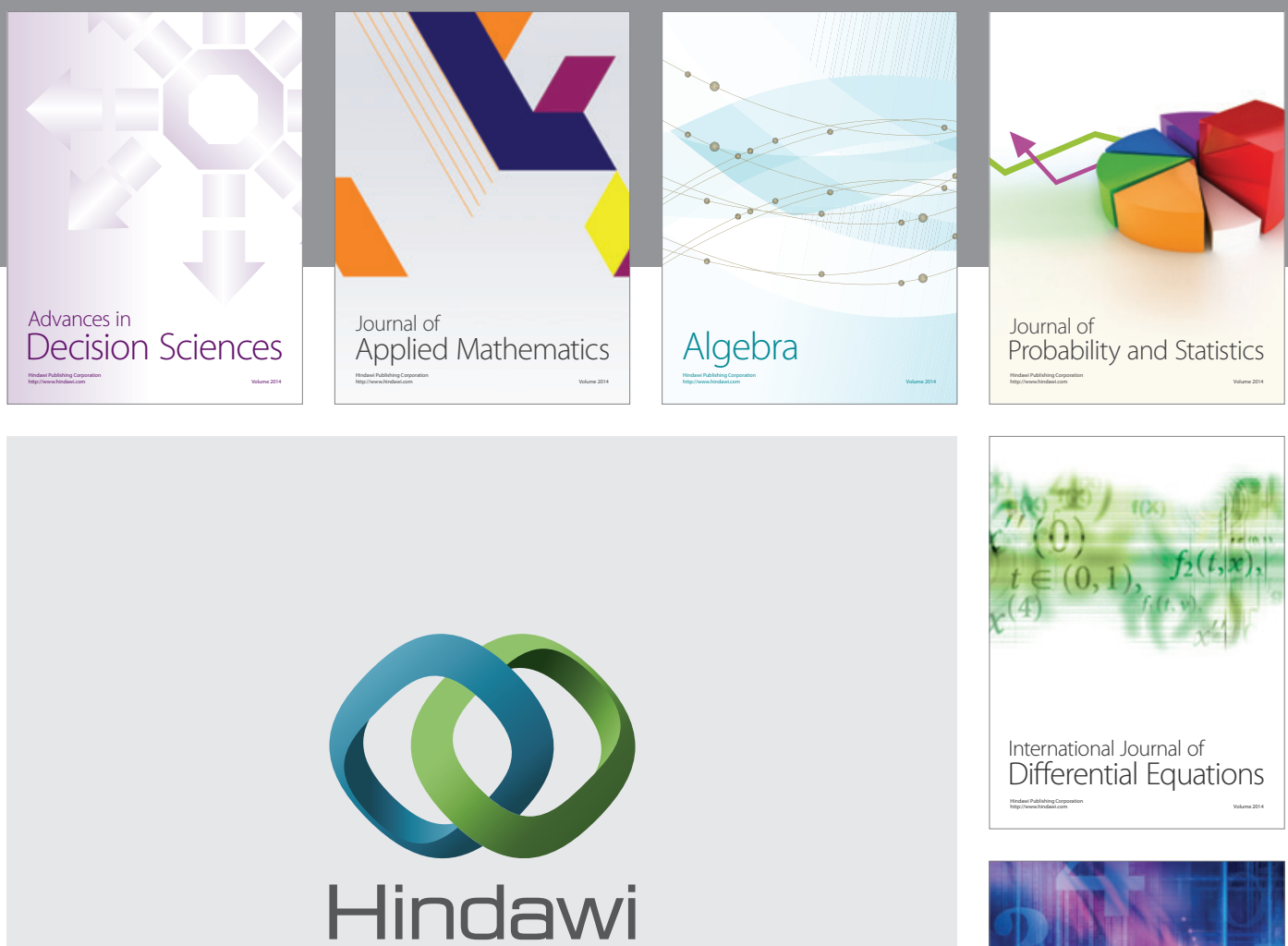

Submit your manuscripts at http://www.hindawi.com
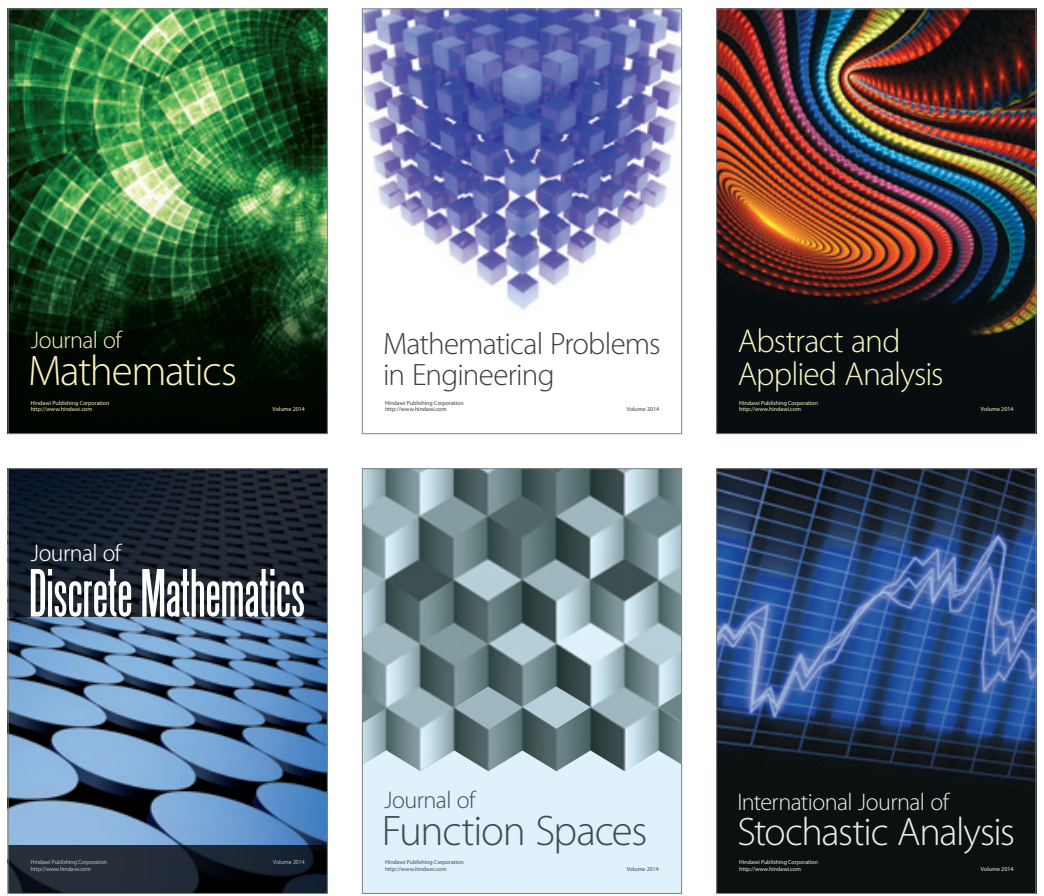

Journal of

Function Spaces

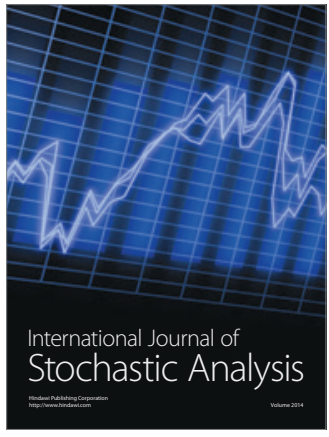

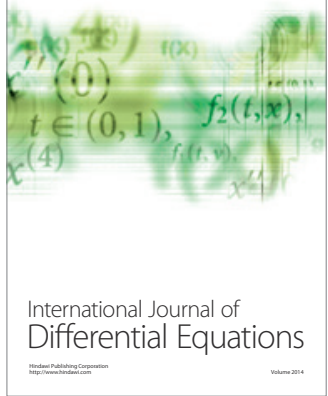
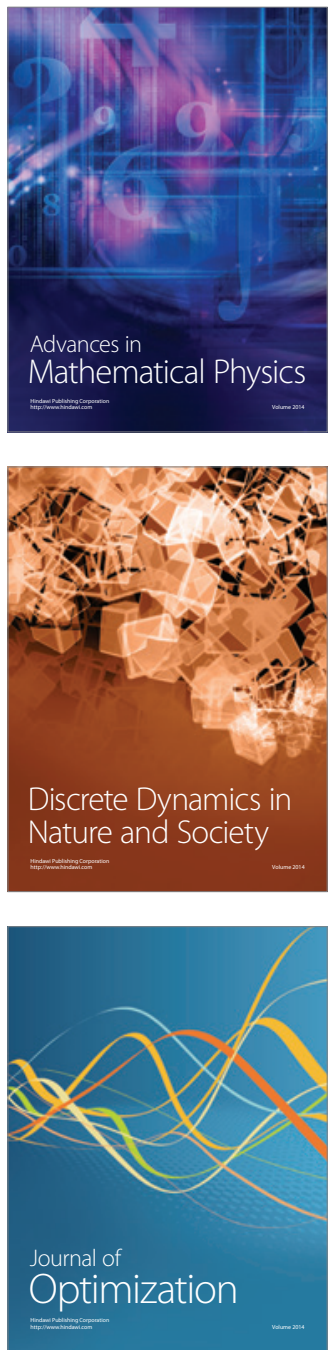